\title{
"Zeroing" in on mathematics in the monkey brain
}

\author{
Michael J. Beran ${ }^{1}$
}

Published online: 22 October 2015

(C) Psychonomic Society, Inc. 2015

\begin{abstract}
Summary A new study documented that monkeys showed selective neuronal responding to the concept of zero during a numerical task, and that there were two distinct classes of neurons that coded the absence of stimuli either through a discrete activation pattern (zero or not zero) or a continuous one for which zero was integrated with other numerosities in the relative rate of activity. These data indicate that monkeys, like humans, have a concept of zero that is part of their analog number line but that also may have unique properties compared to other numerosities.
\end{abstract}

At the heart of all mathematical cognition is a basic sense of numerosity - the idea that concrete and abstract things can be quantified as to their amount, and in a way that allows comparisons, additions, subtractions, and other operations to occur using representations of numerosity. This basic sense of number is ubiquitous in Homo sapiens, present even in societies that have no formal symbolic system for representing numbers (Pica, Lemer, Izard, \& Dehaene, 2004). We have learned that many core aspects of human numerical cognition are shared with other species. Insects, amphibians, fish, birds, and mammals all respond to quantitative stimulus dimensions, allowing them to compare and contrast sets of things. In many of these cases, such judgments and representations of stimuli are truly numerical, and reliant

Michael J. Beran

mjberan@yahoo.com

1 Department of Psychology and Language Research Center, Georgia State University, PO Box 5010, Atlanta, GA 30302, USA heavily on the numbers of items rather than co-occurring features such as amount, density, or surface area. And in a smaller number of cases, the evidence is fairly strong that some species can acquire and use the principles that underlie an important step in mathematical cognition counting. Counting then provides the foundation for more advanced arithmetic, and from there emerge abilities for algebra, geometry, and so forth.

Recent research efforts have extended beyond just behavioral observations of animals making quantitative and numerical judgments to looking at the underlying neural processes that occur during these judgments. In particular, the parietal cortex reflects a critical area in the processing of numbers and other magnitudes by primate species. In some cases comparative research has shown that the same regions activate in human and nonhuman brains during numerical processing of stimuli (for review, see Nieder, 2005).

A fascinating new study by Okuyama, Kuki, and Mushiake (2015) specifically assessed the contributions of parietal lobe activation in monkeys for the concept of zero, and these results further demonstrated continuity in numerical cognition across species. The concept of zero exists even in human societies without formal number notation systems. However, it often emerges slowly in the early acquisition of numerical labeling in children. Zero is a unique kind of number because it represents absence rather than presence, and it may therefore require a different form of representation within the brain compared to number of concepts for presence of stimuli.

Zero as a number concept has not received as much attention in the comparative literature compared to other forms of quantitative stimuli, although when assessed it seems that animals can accommodate nothingness, or zero, as a quantity to be integrated into ordinal sequences or other forms of comparison of sets (e.g., Biro \& 
Matsuzawa, 2001). Okuyama et al. (2015) extended the behavioral assessment of zero to ask whether parietal contributions of the processing of empty sets fit the broader model of numerical representation in monkeys' brains, or somehow looked qualitatively different. They did this by analyzing activity in the ventral intraparietal (VIP) cortex, an area previously shown to have numerosity-specific neurons in single-neuron recording studies with monkeys (e.g., Nieder \& Miller, 2004). The question was whether this region also showed selective activation of neurons that might specifically encode zero relative to other neurons that encoded different numerosities.

In the task (Fig. 1), monkeys were shown a target number of circles on a computer screen. This was the number of items they later had to match. Then, after a brief delay, they were shown a starting quantity onscreen, and they had to use two levers to either add more circles or subtract circles from that initial number to create a match to the target they had seen at trial outset. The monkeys learned this task, although they had more difficulty when larger target quantities were required than smaller ones, as one might expect given what we know about the approximate number system that operates in nonhuman species, young children, and in some cases even adult humans. But, critically for this study, the monkeys were able to accommodate zero sets as targets and as initial set sizes to be modified, in both additive and subtractive trial types.

Okuyama et al. (2015) recorded activity in 614 cells in the VIP and found 185 neurons that showed selective activations to the numerosities 0 to 4 in the target presentation phase and 101 that did so during the delay period before manipulating set size began. Many of these were selectively activated by target zero. Interestingly, this activation suggested that there were two types of "zero" neurons. The first type seemed to exclusively respond to empty sets, as that type did not activate to any other numerosity that was presented. Those neurons showed a rapid buildup in activity throughout the presentation and delay periods, but only when the target contained no circles. The second type of neuron responded to all numerosities, including zero, but showed peak levels of activity to sets of zero and then a gradual decrease of responding to successively larger set sizes. The authors referred to these two types as "exclusive" zero neurons and "continuous" neurons.

So, "zero" fits the analogue number line present in the activity of monkey brains. This could indicate that zero is treated as any other number during tasks for which numerical processing is activated, or it could indicate that these neurons encode presence or absence of to-be-quantified stimuli that, when there is something more than zero, then leads to other number-tuned neurons activating when their target numerosity is present. It will be interesting to examine how zero is encoded and represented in nonhuman primate brains when it is used in other operations, such as being presented as an addend in an arithmetic operation. It also will be interesting to examine how numbers (including zero) presented in non-visual modes activate monkey VIP neurons. One of the key aspects of number concepts is that they can be applied to any kinds of stimuli (concrete or abstract), in any sensory modality, and future work to assess the behavioral and neurobiological breadth and width of nonhuman animals' number sense will provide even greater understanding of the evolutionary emergence of this crucial capacity in our species.

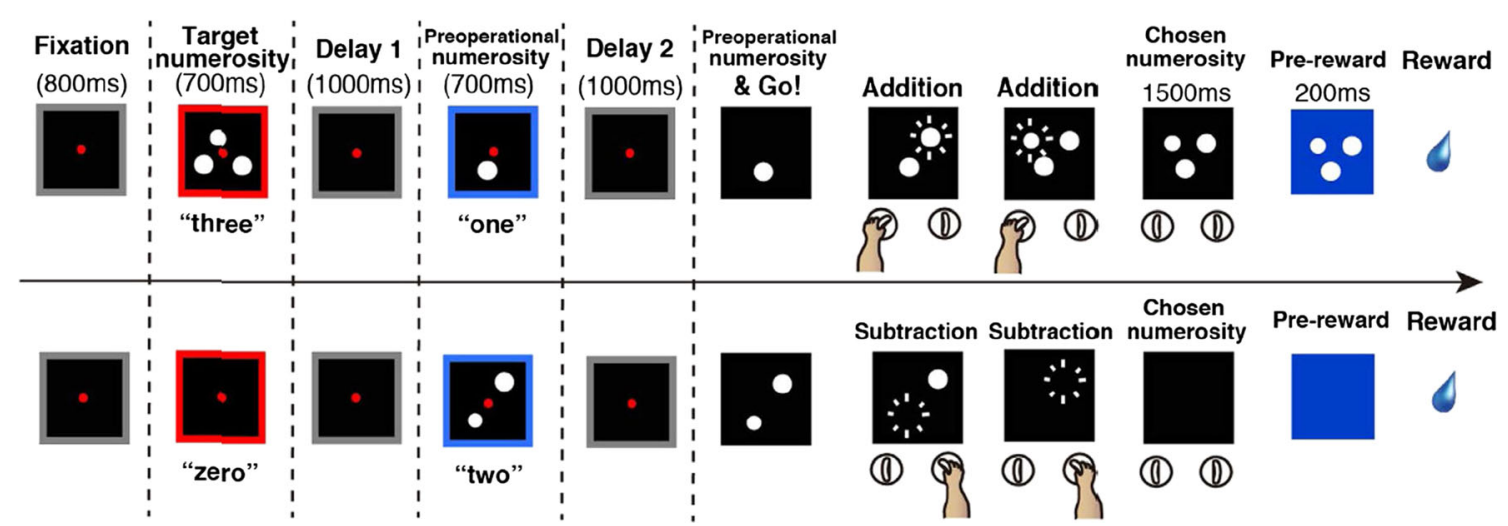

Fig. 1 A schematic representation of the monkey task with a required addition shown in the top row and a required subtraction shown in the bottom row. This task began when the monkey focused on a fixation point. The presentation of a red square containing circles represents the target numerosity. After Delay 1, a blue square containing the second numerosity was displayed as a preoperational numerosity. After Delay 2 , the monkeys were required to remember the target numerosity and manipulate the device for the purpose of matching the preoperational numerosity with the target numerosity. Monkeys were allowed to manipulate either the right or left device, one at a time, as many times as they chose. The number of circles displayed after $1,500 \mathrm{~ms}$ of inactivity was defined as the chosen numerosity. If the chosen numerosity matched the target numerosity, a reward was delivered. This figure and the caption for the figure are taken from Okuyama et al. (2015) 


\section{References}

Biro, D., \& Matsuzawa, T. (2001). Use of numerical symbols by the chimpanzee (Pan troglodytes): Cardinals, ordinals, and the introduction of zero. Animal Cognition, 4, 193-199.

Nieder, A. (2005). Counting on neurons: The neurobiology of numerical competence. Nature Reviews Neuroscience, 6, 177-190.
Nieder, A., \& Miller, E. K. (2004). A parieto-frontal network for visual numerical information in the monkey. Proceedings of the National Academy of Sciences of the United States of America, 101, 7457-7462.

Okuyama, S., Kuki, T., \& Mushiake, H. (2015). Representation of the numerosity "zero" in the parietal cortex of the monkey. Scientific Reports, 5, 10059.

Pica, P., Lemer, C., Izard, V., \& Dehaene, S. (2004). Exact and approximate arithmetic in an Amazonian indigene group. Science, 306, 499-503. 